Report no. 02/13

\title{
Analysis of preconditioners for saddle-point problems
}

\author{
D. Loghin and A. J. Wathen
}

Mixed finite element formulations give rise to large, sparse, block linear systems of equations the solution of which is often sought via a preconditioned iterative technique. In this work we present a general analysis of blockpreconditioners based on the stability conditions inherited from the formulation of the finite element method (the Babuška-Brezzi, or inf-sup conditions).

Oxford University Computing Laboratory

Numerical Analysis Group

Wolfson Building

Parks Road

Oxford, England OX1 3QD

July, 2002 


\section{Contents}

1 Introduction 3

2 Preliminaries 4

2.1 Definitions and standard results . . . . . . . . . . . . . . . . 4

2.2 Norm and field-of-values equivalence . . . . . . . . . . . . . . . 7

2.3 Norm-equivalent preconditioners . . . . . . . . . . . . . . . . . . . 8

$2.4 \quad F O V$-equivalent preconditioners . . . . . . . . . . . . . . 9

3 Saddle-point preconditioners $\quad 10$

3.1 Some useful results . . . . . . . . . . . . . . . . . . . . . . . . 10

3.2 Norm-equivalent preconditioners . . . . . . . . . . . . . . . . . . 13

$3.3 \quad F O V$-equivalent preconditioners . . . . . . . . . . . . . . . 13

4 Applications $\quad 17$

4.1 Example: Navier-Stokes flow . . . . . . . . . . . . . . . . . . . 17

4.2 Numerical experiments . . . . . . . . . . . . . . . . . . . 19

5 Conclusions $\quad 21$ 


\section{Introduction}

Finite element discretizations of partial differential equations yield a sequence of linear systems of equations

$$
K \mathbf{u}=\mathbf{f}
$$

where $K \in \mathbb{R}^{n \times n}$ is a large, sparse matrix and $n \rightarrow \infty$. Moreover, problem (1.1) inherits the stability conditions (the Babuška conditions [6]) required for the finite element method to have a unique solution:

$$
\begin{aligned}
& \max _{\mathbf{w} \in \mathbb{R}^{n} \backslash\{\mathbf{0}\}} \max _{\mathbf{v} \in \mathbb{R}^{n} \backslash\{\mathbf{0}\}} \frac{\mathbf{w}^{t} K \mathbf{v}}{\|\mathbf{w}\|_{H}\|\mathbf{v}\|_{H}} \leq C_{1} \\
& \min _{\mathbf{w} \in \mathbb{R}^{n} \backslash\{\mathbf{0}\}} \max _{\mathbf{v} \in \mathbb{R}^{n} \backslash\{\mathbf{0}\}} \frac{\mathbf{w}^{t} K \mathbf{v}}{\|\mathbf{w}\|_{H}\|\mathbf{v}\|_{H}} \geq C_{2}
\end{aligned}
$$

where $\|\mathbf{v}\|_{H}^{2}=\langle\mathbf{v}, \mathbf{v}\rangle_{H}=\mathbf{v}^{t} H \mathbf{v}$ and $H \in \mathbb{R}^{n \times n}$ is a symmetric and positive-definite matrix defined by the choice of discrete norm on the finite element space.

In this work we are interested in discretizations obtained using mixed finite element formulations, which typically lead to $K$ having a block structure. In particular, we are interested in solving (1.1), under conditions (1.2) and for the case where $K$ has the socalled saddle-point structure

$$
K=\left(\begin{array}{cc}
F & B^{t} \\
B & 0
\end{array}\right)
$$

where $F \in \mathbb{R}^{n_{1} \times n_{1}}$ is nonsingular and generally nonsymmetric and $B \in \mathbb{R}^{n_{2} \times n_{1}}$ has rank $r=n_{2} \leq n_{1}$. We also assume that the norm $\|\cdot\|_{H}$ is given by (or equivalent, independently of $n$, to)

$$
\|\mathbf{v}\|_{H}^{2}=\left\|\mathbf{v}_{1}\right\|_{H_{1}}^{2}+\left\|\mathbf{v}_{2}\right\|_{H_{2}}^{2}
$$

where $\mathbf{v}_{i} \in \mathbb{R}^{n_{i}}$ and $H_{i} \in \mathbb{R}^{n_{i} \times n_{i}}(i=1,2)$ are symmetric and positive-definite matrices.

For this structure of $K$ conditions for existence and uniqueness equivalent to (1.2) were given by Brezzi [9]:

$$
\begin{aligned}
& \max _{\mathbf{q} \in \mathbb{R}^{n_{1}} \backslash\{\mathbf{0}\}} \max _{\mathbf{r} \in \mathbb{R}^{n_{1}} \backslash\{\mathbf{0}\}} \frac{\mathbf{q}^{t} F \mathbf{r}}{\|\mathbf{r}\|_{H_{1}}\|\mathbf{q}\|_{H_{1}}} \leq c_{1} \\
& \max _{\mathbf{q} \in \mathbb{R}^{n_{1}} \backslash\{\mathbf{0}\}} \max _{\mathbf{z} \in \mathbb{R}^{n_{2}} \backslash\{\mathbf{0}\}} \frac{\mathbf{z}^{t} B \mathbf{q}}{\|\mathbf{q}\|_{H_{1}}\|\mathbf{z}\|_{H_{2}}} \leq c_{2} \\
& \min _{\mathbf{r} \in \operatorname{ker} B} \max _{\mathbf{q} \in \operatorname{ker} B} \frac{\mathbf{q}^{t} F \mathbf{r}}{\|\mathbf{r}\|_{H_{1}}\|\mathbf{q}\|_{H_{1}}} \geq c_{3} \\
& \min _{\mathbf{z} \in \mathbb{R}^{n_{2}} \backslash\{\mathbf{0}\}} \max _{\mathbf{q} \in \mathbb{R}^{n_{1}} \backslash\{\mathbf{0}\}} \frac{\mathbf{z}^{t} B \mathbf{q}}{\|\mathbf{q}\|_{H_{1}}\|\mathbf{z}\|_{H_{2}}} \geq c_{4}
\end{aligned}
$$


However, in many applications of interest, condition (1.5c) is satisfied simply because $F$ satisfies the stronger coercivity condition

$$
\max _{\mathbf{r} \in \mathbb{R}^{n_{1}} \backslash\{0\}} \frac{\mathbf{r}^{t} F \mathbf{r}}{\|\mathbf{r}\|_{H_{1}}^{2}} \geq c_{3} .
$$

In this work, we will assume this coercivity condition holds.

When $n$ is large, a competitive alternative to computing directly the solution to (1.1) is to employ an iterative method coupled with a preconditioning technique. More precisely we want to find and characterize preconditioning matrices $P \in \mathbb{R}^{n \times n}$ such that either of the modified systems

$$
P^{-1} K \mathbf{u}=P^{-1} \mathbf{f}, \quad K P^{-1} \widehat{\mathbf{u}}=\mathbf{f} \quad(\widehat{\mathbf{u}}=P \mathbf{u}),
$$

can be solved with an iterative method in a number of iterations independent of $n$. This can be achieved if the preconditioning matrix is equivalent to the system matrix in some sense. We will be concerned with norm and field-of-values equivalence of matrices (see section 2), which provide convergence bounds for iterative methods of Krylov subspace type such as Minimum Residual method (MINRES) or Generalized Minimum Residual method (GMRES).

We note here that preconditioning design and analysis for saddle-point problems has been the subject of active research in a variety of areas of applied mathematics, e.g., groundwater flow [7], [8], [14], [22], [39], [42], Stokes and Navier-Stokes flow [12], [43], [41], [20], [13], [32], [18], [31], [28], [33], [29], elasticity [21],[4], [30], [11], [36], magnetostatics [37], [38] etc. While most results address the symmetric case, non-symmetric preconditioning has also been analyzed for some practical problems. Our contribution provides a general analysis of block-preconditioners for mixed finite element discretizations and describes sufficient conditions for optimal preconditioning.

The paper is structured as follows. Section 2 contains some useful definitions and results. We also define norm and field-of-values equivalence of matrices and outline their relevance to preconditioning techniques for Krylov subspace type methods. The main results of the paper are contained in section 3, where we characterize block-preconditioners for the linear system (1.1) and describe convergence in terms of norms relevant to the finite element setting. Finally, in section 4, we present some examples from the literature.

\section{Preliminaries}

\subsection{Definitions and standard results}

Let $H$ be a symmetric positive-definite matrix. Then $H=H^{1 / 2} H^{1 / 2}$ where $H^{1 / 2}$ is also a symmetric and positive-definite matrix; moreover, $H$ induces a vector-norm $\|\cdot\|_{H}$ with dual-norm $\left(\|\cdot\|_{H}\right)^{D}$ defined as $([27$, p. 275])

$$
\left(\|\mathbf{z}\|_{H}\right)^{D}:=\max _{\mathbf{v} \in \mathbb{R}^{n} \backslash\{\mathbf{0}\}} \frac{\mathbf{v}^{t} \mathbf{z}}{\|\mathbf{v}\|_{H}}=\|\mathbf{z}\|_{H^{-1}} .
$$


Given two symmetric and positive-definite matrices $H_{1} \in \mathbb{R}^{n \times n}, H_{2} \in \mathbb{R}^{m \times m}$ we define the following matrix norm for matrices $M \in \mathbb{R}^{m \times n}$ (see [27, p. 311])

$$
\|M\|_{H_{1}, H_{2}}=\max _{\mathbf{v} \in \mathbb{R}^{n} \backslash\{\mathbf{0}\}} \frac{\|M \mathbf{v}\|_{H_{2}}}{\|\mathbf{v}\|_{H_{1}}}
$$

with the standard notation $\|M\|_{H, H}=\|M\|_{H}$. We highlight here the submultiplicative property of $\|\cdot\|_{H_{1}, H_{2}}$ : if $H_{i} \in \mathbb{R}^{n_{i} \times n_{i}}, i=1,2,3$, are three symmetric and positive-definite matrices and $R \in \mathbb{R}^{n_{1} \times n_{2}}, Q \in \mathbb{R}^{n_{2} \times n_{3}}$ then

$$
\|R Q\|_{H_{3}, H_{1}} \leq\|Q\|_{H_{3}, H_{2}}\|R\|_{H_{2}, H_{1}} .
$$

We will also find useful the following identities

$$
\left\|H_{2}^{-1 / 2} M H_{1}^{-1 / 2}\right\|_{l_{2}}=\|M\|_{H_{1}, H_{2}^{-1}}=\left\|M H_{1}^{-1}\right\|_{H_{1}^{-1}, H_{2}^{-1}}=\left\|H_{2}^{-1} M\right\|_{H_{1}, H_{2}} .
$$

The matrix norms introduced above can be alternatively characterized in the following way.

Lemma 1 Let $M \in \mathbb{R}^{m \times n}$ have full rank and let $H_{1} \in \mathbb{R}^{n \times n}, H_{2} \in \mathbb{R}^{m \times m}$ be two symmetric and positive-definite matrices. Then

$$
\begin{gathered}
\|M\|_{H_{1}, H_{2}^{-1}}=\max _{\mathbf{v} \in \mathbb{R}^{n} \backslash\{\mathbf{0}\}} \max _{\mathbf{w} \in \mathbb{R}^{m} \backslash\{\mathbf{0}\}} \frac{\mathbf{w}^{t} M \mathbf{v}}{\|\mathbf{v}\|_{H_{1}}\|\mathbf{w}\|_{H_{2}}}, \\
\min _{\mathbf{v} \notin \operatorname{ker}(M)} \frac{\|M \mathbf{v}\|_{H_{2}^{-1}}}{\|\mathbf{v}\|_{H_{1}}}=\min _{\mathbf{v} \notin \operatorname{ker}(M)} \max _{\mathbf{w} \in \mathbb{R}^{m} \backslash\{\mathbf{0}\}} \frac{\mathbf{w}^{t} M \mathbf{v}}{\|\mathbf{v}\|_{H_{1}}\|\mathbf{w}\|_{H_{2}}} .
\end{gathered}
$$

Hence, if $n=m$,

$$
\left\|M^{-1}\right\|_{H_{2}^{-1}, H_{1}}^{-1}=\min _{\mathbf{v} \in \mathbb{R}^{n} \backslash\{\mathbf{0}\}} \max _{\mathbf{w} \in \mathbb{R}^{n} \backslash\{\mathbf{0}\}} \frac{\mathbf{w}^{t} M \mathbf{v}}{\|\mathbf{v}\|_{H_{1}}\|\mathbf{w}\|_{H_{2}}} .
$$

Proof These results are generalizations of the results in [10]. However, we include the proofs here for completeness. We have

$$
\begin{aligned}
\|M\|_{H_{1}, H_{2}^{-1}} & =\max _{\mathbf{v} \in \mathbb{R}^{n} \backslash\{\mathbf{0}\}} \frac{\|M \mathbf{v}\|_{H_{2}^{-1}}}{\|\mathbf{v}\|_{H_{1}}} \\
& =\max _{\mathbf{v} \in \mathbb{R}^{n} \backslash\{\mathbf{0}\}}\left\{\frac{1}{\|\mathbf{v}\|_{H_{1}}} \max _{\mathbf{w} \in \mathbb{R}^{m} \backslash\{\mathbf{0}\}} \frac{\mathbf{w}^{t} M \mathbf{v}}{\|\mathbf{w}\|_{H_{2}}}\right\} \\
& =\max _{\mathbf{v} \in \mathbb{R}^{n} \backslash\{\mathbf{0}\}} \max _{\mathbf{w} \in \mathbb{R}^{m} \backslash\{\mathbf{0}\}} \frac{\mathbf{w}^{t} M \mathbf{v}}{\|\mathbf{v}\|_{H_{1}}\|\mathbf{w}\|_{H_{2}}} .
\end{aligned}
$$

For the second equality we have

$$
\begin{aligned}
\min _{\mathbf{v} \notin \operatorname{ker}(M)} \frac{\|M \mathbf{v}\|_{H_{2}^{-1}}}{\|\mathbf{v}\|_{H_{1}}} & =\min _{\mathbf{v} \notin \operatorname{ker}(M)}\left\{\frac{1}{\|\mathbf{v}\|_{H_{1}}} \max _{\mathbf{w} \in \mathbb{R}^{m} \backslash\{\mathbf{0}\}} \frac{\mathbf{w}^{t} M \mathbf{v}}{\|\mathbf{w}\|_{H_{2}}}\right\} \\
& =\min _{\mathbf{v} \notin \operatorname{ker}(M)} \max _{\mathbf{w} \in \mathbb{R}^{m} \backslash\{\mathbf{0}\}} \frac{\mathbf{w}^{t} M \mathbf{v}}{\|\mathbf{v}\|_{H_{1}}\|\mathbf{w}\|_{H_{2}}} .
\end{aligned}
$$


Hence, when $n=m, M$ is invertible with $\operatorname{ker}(M)=\{\mathbf{0}\}$ and the left-hand side in the above equality becomes

$$
\begin{aligned}
\min _{\mathbf{v} \in \mathbb{R}^{n} \backslash\{\mathbf{0}\}} \frac{\|M \mathbf{v}\|_{H_{2}^{-1}}}{\|\mathbf{v}\|_{H_{1}}} & =\min _{\mathbf{z} \in \mathbb{R}^{n} \backslash\{\mathbf{0}\}} \frac{\|\mathbf{z}\|_{H_{2}^{-1}}}{\left\|M^{-1} \mathbf{z}\right\|_{H_{1}}} \quad(\mathbf{z}=M \mathbf{v}) \\
& =\left(\max _{\mathbf{z} \in \mathbb{R}^{n} \backslash\{\mathbf{0}\}} \frac{\left\|M^{-1} \mathbf{z}\right\|_{H_{1}}}{\|\mathbf{z}\|_{H_{2}^{-1}}}\right)^{-1} \\
& =\left\|M^{-1}\right\|_{H_{2}^{-1}, H_{1}}^{-1}
\end{aligned}
$$

which yields the last equality.

Note that $\|M\|_{H_{1}, H_{2}^{-1}}=\left\|M^{t}\right\|_{H_{2}, H_{1}^{-1}}$ since

$$
\begin{aligned}
\max _{\mathbf{v} \in \mathbb{R}^{n} \backslash\{\mathbf{0}\}} \max _{\mathbf{w} \in \mathbb{R}^{m} \backslash\{\mathbf{0}\}} \frac{\mathbf{w}^{t} M \mathbf{v}}{\|\mathbf{v}\|_{H_{1}}\|\mathbf{w}\|_{H_{2}}} & =\max _{\mathbf{v} \in \mathbb{R}^{n} \backslash\{\mathbf{0}\}} \max _{\mathbf{w} \in \mathbb{R}^{m} \backslash\{\mathbf{0}\}} \frac{\mathbf{v}^{t} M^{t} \mathbf{w}}{\|\mathbf{v}\|_{H_{1}}\|\mathbf{w}\|_{H_{2}}} \\
& =\max _{\mathbf{w} \in \mathbb{R}^{m} \backslash\{\mathbf{0}\}}\left\{\frac{1}{\|\mathbf{w}\|_{H_{2}}} \max _{\mathbf{v} \in \mathbb{R}^{n} \backslash\{\mathbf{0}\}} \frac{\mathbf{v}^{t}\left(M^{t} \mathbf{w}\right)}{\|\mathbf{v}\|_{H_{1}}}\right\} \\
& =\max _{\mathbf{w} \in \mathbb{R}^{m} \backslash\{\mathbf{0}\}} \frac{\left\|M^{t} \mathbf{w}\right\|_{H_{1}^{-1}}}{\|\mathbf{w}\|_{H_{2}}} \\
& =\left\|M^{t}\right\|_{H_{2}, H_{1}^{-1}}
\end{aligned}
$$

We also recall here the definition of the field of values and some standard related results.

Definition 1 Let $M, H \in \mathbb{R}^{n \times n}$, with $H$ symmetric and positive definite. The $H$-field of values of the matrix $M$, denote by $\mathcal{W}_{H}(M)$, is a set in the complex plane given by

$$
\mathcal{W}_{H}(M)=\left\{\mathbf{x} \in \mathbb{C}: \frac{\mathbf{x}^{*} H M \mathbf{x}}{\mathbf{x}^{*} H \mathbf{x}}\right\} .
$$

When $H=I$, the set is called the field of values and is denoted by $\mathcal{W}(M)$.

The following properties are useful.

Lemma 2 Let $M, H \in \mathbb{R}^{n \times n}$, with $H$ symmetric and positive definite. The following holds for all $H$ :

(i) The eigenvalues of $M$ are contained in $\mathcal{W}_{H}(M)$.

(ii) For all $z \in \mathcal{W}_{H}(M)$,

$$
\min _{z} \operatorname{Re} z=\min _{\mathbf{x} \in \mathbb{R}^{n}\{\mathbf{0}\}} \frac{\mathbf{x}^{t} H M \mathbf{x}}{\mathbf{x}^{t} H \mathbf{x}} \leq\left\|M^{-1}\right\|_{H}^{-1}, \quad|z| \leq\|M\|_{H}
$$




\subsection{Norm and field-of-values equivalence}

The following notions of equivalence between matrices are used later to define optimality of preconditioners.

Definition $2 H$-norm equivalence Non-singular matrices $A, B \in \mathbb{R}^{n \times n}$ are said to be $H$ norm equivalent if there exist constants $\gamma, \Gamma$ independent of $n$ such that for all $\mathbf{x} \in \mathbb{R}^{n} \backslash\{\mathbf{0}\}$

$$
\gamma \leq \frac{\|A \mathbf{x}\|_{H}}{\|B \mathbf{x}\|_{H}} \leq \Gamma
$$

We write

$$
A \sim_{H} B
$$

Another characterization of $H$-norm equivalence is given by the following lemmata.

Lemma $3 A \sim_{H} B$ if and only if $\left\|A B^{-1}\right\|_{H} \leq \Gamma$ and $\left\|B A^{-1}\right\|_{H} \leq \gamma^{-1}$.

Definition 3 Field-of-values ( $F O V$ ) equivalence Non-singular matrices $A, B \in \mathbb{R}^{n \times n}$ are said to be $F O V$-equivalent if there exist constants $\gamma, \Gamma$ independent of $n$ such that for all $\mathbf{x} \in \mathbb{R}^{n} \backslash\{\mathbf{0}\}$

$$
\gamma \leq \frac{\left\langle\mathbf{x}, A B^{-1} \mathbf{x}\right\rangle_{H}}{\langle\mathbf{x}, \mathbf{x}\rangle_{H}}, \quad \frac{\left\|A B^{-1} \mathbf{x}\right\|_{H}}{\|\mathbf{x}\|_{H}} \leq \Gamma
$$

We write

$$
A \approx_{H} B
$$

Remark 1 The FOV-equivalence simply implies that the $H$-field of values of $A B^{-1}$ is in the right half-plane and is bounded independently of $n$. We note that if $H=I$ and $A, B$ are symmetric and positive-definite, the FOV-equivalence becomes the well-known spectral equivalence introduced in [19]. This is a consequence of the fact that the field of values of a real symmetric matrix is the convex hull of its spectrum.

Remark 2 Both $H$-norm equivalence and FOV-equivalence imply that (cf. Lemma 2)

$$
\gamma<\left|\lambda\left(A B^{-1}\right)\right|<\Gamma
$$

where $\lambda\left(A B^{-1}\right)$ are the eigenvalues of $A B^{-1}$. However, FOV-equivalence is a stronger statement: if $A \approx_{H} B$ then $A \sim_{H} B$. On the other hand, it is easy to see that $\sim_{H}$ is an equivalence relation on $\mathbb{R}^{n \times n}$ while $\approx_{H}$ is not even reflexive.

Both equivalences can be shown to be descriptive in terms of convergence behaviour of iterative methods with preconditioning. In particular, they can be used to describe convergence of the following two algorithms. 
Algorithm 1 : Minimum Residual (MINRES). If $A \sim_{H} B$ and $A B^{-1}$ is symmetric and indefinite with respect to $\langle\cdot, \cdot\rangle_{H}$ the MINRES algorithm applied to $A$ with right preconditioner $B$ converges in a number of iterations independent of $n$. Moreover, the residuals satisfy [26, Eqn (3.14)]

$$
\frac{\left\|\mathbf{r}^{k}\right\|_{H}}{\left\|\mathbf{r}^{0}\right\|_{H}} \leq 2\left(\frac{\Gamma-\gamma}{\Gamma+\gamma}\right)^{k / 2},
$$

where $\gamma, \Gamma$ are the constants in Definition 2, which are related to the bounds on the eigenvalues as given in Remark 2.

Algorithm 2 : Generalized Minimum Residual (GMRES). If $A \approx_{H} B$ the $G M$ $R E S$ algorithm converges with respect to $\langle\cdot, \cdot\rangle_{H}$ in a number of iterations independent of $n$. Moreover, the residuals satisfy [15], [40, Thm 6.7]

$$
\frac{\left\|\mathbf{r}^{k}\right\|_{H}}{\left\|\mathbf{r}^{0}\right\|_{H}} \leq\left(1-\frac{\gamma^{2}}{\Gamma^{2}}\right)^{k / 2},
$$

where $\gamma, \Gamma$ are the constants in Definition 3.

Remark 3 It is well-known that the bounds on the convergence of MINRES and GMRES can be shown to be pessimistic in certain applications. In such situations, they still describe convergence independent of $n$, albeit in an asymptotic sense.

In the following, we will establish sufficient conditions for norm and $F O V$-equivalence with respect to the $H$-norm for left preconditioning, and $H^{-1}$-norm for right preconditioning. Since left preconditioning changes the residuals (and thus the norm) in the iteration, while right preconditioning leaves them unchanged, the question arises as to what norm to consider for convergence. It can be shown that the natural norm for convergence for finite element problems is $\|\cdot\|_{H^{-1}}$, and that left preconditioning also leads to minimization with respect to this norm, provided the preconditioner is norm-equivalent to the matrix norm. These issues are considered in [25], [24], [35], [1] for symmetric problems and in [3], [2] for nonsymmetric problems.

We end this section with some useful characterizations of preconditioners in terms of norm and $F O V$-equivalence, for problems for which (1.2) are satisfied.

\subsection{Norm-equivalent preconditioners}

Norm-equivalence is inherent in the stability requirements (1.2), as the following result demonstrates.

Lemma 4 Let (1.2) hold. Then

$$
H \sim_{H^{-1}} K, \quad H^{-1} \sim_{H} K^{-1} .
$$

In particular,

$$
\begin{aligned}
\left\|H^{-1} K\right\|_{H} & =\left\|K H^{-1}\right\|_{H^{-1}} \leq C_{1}, \\
\left\|K^{-1} H\right\|_{H} & =\left\|H K^{-1}\right\|_{H^{-1}} \leq C_{2}^{-1} .
\end{aligned}
$$


Proof Using (1.2a) and (2.5) we get

$$
\begin{aligned}
C_{1} & \geq \max _{\mathbf{v}, \mathbf{w} \in \mathbb{R}^{n} \backslash\{\mathbf{0}\}} \frac{\mathbf{w}^{t} K \mathbf{v}}{\|\mathbf{w}\|_{H}\|\mathbf{v}\|_{H}} \\
& =\|K\|_{H, H^{-1}}
\end{aligned}
$$

and bound (2.10a) follows from (2.4). Likewise, (1.2b) and (2.7) give

$$
\begin{aligned}
C_{2} & \leq \min _{\mathbf{v} \in \mathbb{R}^{n} \backslash\{\mathbf{0}\}} \max _{\mathbf{w} \in \mathbb{R}^{n} \backslash\{\mathbf{0}\}} \frac{\mathbf{w}^{t} K \mathbf{v}}{\|\mathbf{v}\|_{H}\|\mathbf{w}\|_{H}} \\
& =\left(\left\|K^{-1}\right\|_{H^{-1}, H}\right)^{-1}
\end{aligned}
$$

and $(2.10 \mathrm{~b})$ follows similarly.

The previous result can be used to characterize a general class of preconditioners.

Theorem 5 Let (1.2) hold and assume $P \in \mathbb{R}^{n \times n}$ satisfies

$$
P \sim_{H^{-1}} H
$$

Then

$$
P \sim_{H^{-1}} K, \quad P^{-1} \sim_{H} K^{-1} .
$$

Proof Since $P \sim_{H^{-1}} H$ is equivalent to $P^{-1} \sim_{H} H^{-1}$, the result follows from the transitivity property of $\sim_{H}$ and $\sim_{H^{-1}}$ and Lemma 4 .

Remark 4 The result of Lemma 4 establishes mesh-independent bounds on the condition number of $H^{-1} K$ and $K^{-1}$ with respect to the natural norm of the problem and its dual. Theorem 5 states that if $P$ and $H$ are $H$-equivalent then $P$ will be a useful left or right preconditioner for $K$ in a suitable norm.

\section{$2.4 \quad F O V$-equivalent preconditioners}

If requirement $(1.2 \mathrm{~b})$ is replaced by the stronger relation

$$
\min _{\mathbf{v} \in \mathbb{R}^{n} \backslash\{\mathbf{0}\}} \frac{\mathbf{v}^{t} K \mathbf{v}}{\|\mathbf{v}\|_{H}^{2}} \geq C_{3},
$$

we can prove similar statements about $F O V$-equivalence. We note here that this condition arises quite naturally in mixed finite element formulations and is not a stringent requirement.

Theorem 6 Let (1.2a), (2.11) hold. Then

$$
K \approx_{H^{-1}} H .
$$

Proof We have

$$
\frac{\left\langle\mathbf{x}, K H^{-1} \mathbf{x}\right\rangle_{H^{-1}}}{\langle\mathbf{x}, \mathbf{x}\rangle_{H^{-1}}}=\frac{\mathbf{x}^{t} K \mathbf{x}}{\mathbf{x}^{t} H \mathbf{x}} \geq C_{3} \quad(\operatorname{using}(2.11))
$$

and $\left\|K H^{-1}\right\|_{H^{-1}}<C_{1}$ (cf. proof of Lemma 4). Thus, $K \approx_{H^{-1}} H$. 


\section{Saddle-point preconditioners}

In the following we consider saddle-point problems for which $K$ has the block structure

$$
K=\left(\begin{array}{cc}
F & B^{t} \\
B & 0
\end{array}\right)
$$

and conditions (1.2) are satisfied and where $F$ is in general non-symmetric. The norm $\|\cdot\|_{H}: \mathbb{R}^{n}$ is defined as in (1.4) and defines the symmetric and positive-definite matrix

$$
H=\left(\begin{array}{cc}
H_{1} & 0 \\
0 & H_{2}
\end{array}\right)
$$

Before we prove the main results we need to establish the following results.

\subsection{Some useful results}

In the following we denote by $S$ the Schur complement of $F$ in $K$

$$
S=B F^{-1} B^{t}
$$

Lemma 7 Let (1.2) hold. Then

$$
\|F\|_{H_{1}, H_{1}^{-1}} \leq C_{1}, \quad\|B\|_{H_{1}, H_{2}^{-1}} \leq C_{1}, \quad\left\|S^{-1}\right\|_{H_{2}^{-1}, H_{2}} \leq C_{2}^{-1}
$$

and

$$
\min _{\mathbf{q} \in \mathbb{R}^{n_{2}} \backslash\{\mathbf{0}\}} \frac{\left\|B^{t} \mathbf{q}\right\|_{H_{1}^{-1}}}{\|\mathbf{q}\|_{H_{2}}}=\min _{\mathbf{q} \in \mathbb{R}^{n_{2}} \backslash\{\mathbf{0}\}} \max _{\mathbf{z} \in \mathbb{R}^{n_{1}} \backslash\{\mathbf{0}\}} \frac{\mathbf{q}^{t} B \mathbf{z}}{\|\mathbf{z}\|_{H_{1}}\|\mathbf{q}\|_{H_{2}}} \geq C_{2} .
$$

Proof From (2.4) with $H=H_{1}=H_{2}, M=K$ and Lemma 4, $\left\|H^{-1 / 2} K H^{-1 / 2}\right\|_{l_{2}}=$ $\left\|H^{-1} K\right\|_{H} \leq C_{1}$ from which it follows that the $l_{2}-$ norms of each block of

$$
H^{-1 / 2} K H^{-1 / 2}=\left(\begin{array}{cc}
H_{1}^{-1 / 2} F H_{1}^{-1 / 2} & H_{1}^{-1 / 2} B^{t} H_{2}^{-1 / 2} \\
H_{2}^{-1 / 2} B H_{1}^{-1 / 2} & 0
\end{array}\right)
$$

are bounded by $C_{1}$ and the bounds on $\|F\|_{H_{1}, H_{1}^{-1}}$ and $\|B\|_{H_{1}, H_{2}^{-1}}$ follow using (2.4). Likewise, $\left\|H^{1 / 2} K^{-1} H^{1 / 2}\right\|_{l_{2}} \leq C_{2}^{-1}$ and the bound on the Schur complement follows similarly by noting that the inverse of $K$ is given by

$$
K^{-1}=\left(\begin{array}{cc}
F^{-1}\left(F-B^{t} S^{-1} B\right) F^{-1} & F^{-1} B^{t} S^{-1} \\
S^{-1} B F^{-1} & -S^{-1}
\end{array}\right) .
$$

Finally, the last bound follows from (1.2b) and Lemma 1. Since for all $\mathbf{v} \in \mathbb{R}^{n} \backslash\{\mathbf{0}\}$

$$
C_{2} \leq \max _{\mathbf{w} \in \mathbb{R}^{n} \backslash\{\mathbf{0}\}} \frac{\mathbf{w}^{t} K \mathbf{v}}{\|\mathbf{v}\|_{H}\|\mathbf{w}\|_{H}}
$$


the choice $\mathbf{v}^{t}=\left(0, \mathbf{q}^{t}\right)$ yields after setting $\mathbf{w}^{t}=\left(\mathbf{z}^{t}, \mathbf{r}^{t}\right)$

$$
\begin{aligned}
C_{2} & \leq \max _{\mathbf{z}, \mathbf{r}} \frac{\mathbf{z}^{t} B^{t} \mathbf{q}}{\left(\|\mathbf{z}\|_{H_{1}}^{2}+\|\mathbf{r}\|_{H_{2}}^{2}\right)^{1 / 2}\|\mathbf{q}\|_{H_{2}}} \\
& =\max _{\mathbf{z} \in \mathbb{R}^{n_{1}} \backslash\{\mathbf{0}\}} \frac{\mathbf{z}^{t} B^{t} \mathbf{q}}{\|\mathbf{z}\|_{H_{1}}\|\mathbf{q}\|_{H_{2}}}
\end{aligned}
$$

for all $\mathbf{q} \in \mathbb{R}^{n_{2}} \backslash\{\mathbf{0}\}$.

Lemma 8 Let (1.2) hold. If there exists a constant $C_{3}$ independent of $n$ such that

$$
\mathbf{x}^{t} F \mathbf{x} \geq C_{3}\|\mathbf{x}\|_{H_{1}}^{2} \quad \forall \mathbf{x} \in \mathbb{R}^{n_{1} \times n_{1}}
$$

then

$$
S \approx_{H_{2}^{-1}} H_{2} \text {. }
$$

Proof Since Lemma 7 provides the bound

$$
\left\|H_{2} S^{-1}\right\|_{H_{2}^{-1}}=\left\|S^{-1}\right\|_{H_{2}^{-1}, H_{2}} \leq C_{2}^{-1}
$$

we only need to find a bound on

$$
\frac{\left\langle\mathbf{x}, S H_{2}^{-1} \mathbf{x}\right\rangle_{H_{2}^{-1}}}{\langle\mathbf{x}, \mathbf{x}\rangle_{H_{2}^{-1}}}=\frac{\mathbf{x}^{t} S \mathbf{x}}{\mathbf{x}^{t} H_{2} \mathbf{x}}
$$

We have

$$
\begin{aligned}
\min _{\mathbf{x} \in \mathbb{R}^{n_{2}} \backslash\{\mathbf{0}\}} \frac{\mathbf{x}^{t} B F^{-1} B^{t} \mathbf{x}}{\mathbf{x}^{t} H_{2} \mathbf{x}} & =\min _{\mathbf{x} \in \mathbb{R}^{n_{2}} \backslash\{\mathbf{0}\}} \frac{\mathbf{x}^{t} B F^{-1} B^{t} \mathbf{x}}{\mathbf{x}^{t} B H_{1}^{-1} B^{t} \mathbf{x}} \frac{\mathbf{x}^{t} B H_{1}^{-1} B^{t} \mathbf{x}}{\mathbf{x}^{t} H_{2} \mathbf{x}} \\
& \geq \min _{\mathbf{y} \in \mathbb{R}^{n_{2}} \backslash\{\mathbf{0}\}} \frac{\mathbf{y}^{t} F^{-1} \mathbf{y}}{\mathbf{y}^{t} H_{1}^{-1} \mathbf{y}} \min _{\mathbf{x} \in \mathbb{R}^{n_{2}} \backslash\{\mathbf{0}\}} \frac{\left\|B^{t} \mathbf{x}\right\|_{H_{1}^{-1}}^{2}}{\|\mathbf{x}\|_{H_{2}}^{2}} \\
& \geq C_{2}^{2} \min _{\mathbf{y} \in \mathbb{R}^{n_{2}} \backslash\{\mathbf{0}\}} \frac{\mathbf{y}^{t} F^{-1} \mathbf{y}}{\mathbf{y}^{t} H_{1}^{-1} \mathbf{y}},
\end{aligned}
$$

Now, (1.2) imply (1.5a) which together with (3.2) implies that $F \approx_{H_{1}^{-1}} H_{1}$. Let $H_{F}=\left(F+F^{t}\right) / 2, S_{F}=\left(F-F^{t}\right) / 2$. Since $\mathbf{x}^{t} F \mathbf{x}=\mathbf{x}^{t} H_{F} \mathbf{x}$, the $F O V$-equivalence of $F$ and $H_{1}$ implies that

$$
C_{3} \leq \frac{\mathbf{x}^{t} H_{F} \mathbf{x}}{\mathbf{x}^{t} H_{1} \mathbf{x}} \leq C_{1}
$$

Hence

$$
\frac{\mathbf{y}^{t} F^{-1} \mathbf{y}}{\mathbf{y}^{t} H_{1}^{-1} \mathbf{y}}=\frac{\mathbf{y}^{t} F^{-1} \mathbf{y}}{\mathbf{y}^{t} H_{F}^{-1} \mathbf{y}} \cdot \frac{\mathbf{y}^{t} H_{F}^{-1} \mathbf{y}}{\mathbf{y}^{t} H_{1}^{-1} \mathbf{y}} \geq C_{1}^{-1} \min _{\mathbf{y} \in \mathbb{R}^{n} \backslash\{\mathbf{0}\}} \frac{\mathbf{y}^{t} F^{-1} \mathbf{y}}{\mathbf{y}^{t} H_{F}^{-1} \mathbf{y}} \geq C_{1}^{-1} \min _{\mathbf{z} \in \mathbb{R}^{n} \backslash\{\mathbf{0}\}} \frac{\mathbf{z}^{t} \tilde{F}^{-1} \mathbf{z}}{\mathbf{z}^{t} \mathbf{z}}
$$

where $\tilde{F}=I+\tilde{N}, \tilde{N}=H_{F}^{-1 / 2} S_{F} H_{F}^{-1 / 2}$. Since $\tilde{F}$ (and thus $\tilde{F}^{-1}$ ) is a normal matrix, its field of values is the convex hull of its eigenvalues ([27, p. 11]). Hence,

$$
\min _{\mathbf{y} \in \mathbb{R}^{n} \backslash\{\mathbf{0}\}} \frac{\mathbf{y}^{t} \tilde{F}^{-1} \mathbf{y}}{\mathbf{y}^{t} \mathbf{y}}=\min _{k} \operatorname{Re} \frac{1}{\lambda_{k}(\tilde{F})}=\min _{k} \operatorname{Re} \frac{1}{1+\lambda_{k}(\tilde{N})}=\frac{1}{\max _{k}\left|\lambda_{k}(\tilde{F})\right|^{2}}
$$


and since

$\max _{k}\left|\lambda_{k}(\tilde{F})\right| \leq\|\tilde{F}\|_{l_{2}}=\left\|H_{F}^{-1 / 2} F H_{F}^{-1 / 2}\right\|_{l_{2}} \leq\left\|H_{1}{ }^{-1 / 2} F H_{1}{ }^{-1 / 2}\right\|_{l_{2}} \kappa_{2}\left(H_{F}^{-1 / 2} H_{1}{ }^{1 / 2}\right) \leq C_{1}\left(\frac{C_{1}}{C_{3}}\right)^{1 / 2}$

the result follows

$$
\frac{\left\langle\mathbf{x}, S H_{2}^{-1} \mathbf{x}\right\rangle_{H_{2}^{-1}}}{\langle\mathbf{x}, \mathbf{x}\rangle_{H_{2}^{-1}}} \geq C_{3} C_{2}^{2} / C_{1}^{4},
$$

and the proof is complete.

Remark 5 The last bound in the previous lemma can be improved to $C_{2}^{2} / C_{1}^{2}$ if the matrix $H_{1}$ is the symmetric part of $F: H_{1}=H_{F}$. This situation is common and the bound can be shown to be tight in some applications (see section 4).

A natural question to ask is under what conditions the equivalence of the Schur complement $S$ to the norm $H_{2}$ is preserved when $F$ is replaced with some approximation $\widetilde{F}$. The answer is provided below.

Lemma 9 Let $(1.2)$ hold, and let $\widetilde{F} \approx_{H_{1}^{-1}} H_{1}$. Then $\widetilde{S}=B \widetilde{F}^{-1} B^{t}$ satisfies

$$
\widetilde{S} \approx_{H_{2}^{-1}} H_{2}
$$

Proof Since (1.2) hold, conditions (1.5b), (1.5d) will hold. On the other hand, $\widetilde{F} \approx_{H_{1}^{-1}} H_{1}$, and thus (1.5a) and (1.6) (and therefore (1.5c)) will hold for $\widetilde{F}$. Hence the stability conditions (1.5) are satisfied for matrices $\widetilde{F}, B$. It follows that

$$
\widetilde{K}=\left(\begin{array}{cc}
\widetilde{F} & B^{t} \\
B & 0
\end{array}\right)
$$

satisfies (1.2). Hence, the results of Lemma 8 apply with $F$ replaced by $\widetilde{F}$.

In the following discussion, we will consider three preconditioners based on the structure of $K$ :

$$
P_{C}=\left(\begin{array}{cc}
P_{1} & 0 \\
0 & P_{2}
\end{array}\right), \quad P_{L}=\left(\begin{array}{cc}
P_{1} & 0 \\
B & P_{2}
\end{array}\right), \quad P_{R}=\left(\begin{array}{cc}
P_{1} & B^{t} \\
0 & P_{2}
\end{array}\right)
$$

We are interested in finding necessary and sufficient conditions for these preconditioners to lead to solution algorithms with convergence independent of $n$. More precisely, we would like to characterize norm and $F O V$-equivalence to $K$ of our preconditioners. We remark here that we only need describe norm-equivalence to $H$ of our preconditioners (cf. Theorem $5)$. 


\subsection{Norm-equivalent preconditioners}

The characterization of norm-equivalence for the above preconditioners is straightforward.

Theorem 10 Let (1.2) hold and let $P \in\left\{P_{C}, P_{L}, P_{R}\right\}$. Then $P \sim_{H^{-1}} K$ if

$$
P_{1} \sim_{H_{1}^{-1}} H_{1} \quad \text { and } \quad P_{2} \sim_{H_{2}^{-1}} H_{2} .
$$

Proof By Lemma 4, $K \sim_{H^{-1}} H$. Hence, by the transitivity of $\sim_{H^{-1}}$ we only need to show $P \sim_{H^{-1}} H$. Since the case $P=P_{C}$ is trivial, we will prove the result for $P_{L}$ first.

Assume that $P_{i} \sim_{H_{i}^{-1}} H_{i}, i=1,2$, i.e. that

$$
\left\|H_{i}^{-1} P_{i}\right\|_{H_{i}} \leq \Gamma_{i}, \quad\left\|P_{i}^{-1} H_{i}\right\|_{H_{i}} \leq \gamma_{i}, \quad i=1,2 .
$$

Since

$$
H^{-1 / 2} P_{L} H^{-1 / 2}=\left(\begin{array}{cc}
H_{1}^{-1 / 2} P_{1} H_{1}^{-1 / 2} & 0 \\
H_{2}^{-1 / 2} B H_{1}^{-1 / 2} & H_{2}^{-1 / 2} P_{2} H_{2}^{-1 / 2}
\end{array}\right)
$$

we get using Lemma 7 and the equivalence $P_{i} \sim_{H_{i}^{-1}} H_{i}, i=1,2$,

$$
\begin{aligned}
\left\|H^{-1 / 2} P_{L} H^{-1 / 2}\right\|_{l_{2}} & \leq\left\|H_{1}^{-1 / 2} P_{1} H_{1}^{-1 / 2}\right\|_{l_{2}}+\left\|H_{2}^{-1 / 2} B H_{1}^{-1 / 2}\right\|_{l_{2}}+\left\|H_{2}^{-1 / 2} P_{2} H_{2}^{-1 / 2}\right\|_{l_{2}} \\
& =\left\|H_{1}^{-1} P_{1}\right\|_{H_{1}}+\|B\|_{H_{1}, H_{2}^{-1}}+\left\|H_{2}^{-1} P_{2}\right\|_{H_{2}} \\
& \leq \Gamma_{1}+C_{1}+\Gamma_{2} .
\end{aligned}
$$

Similarly, since

$$
\begin{aligned}
& H^{1 / 2} P_{L}^{-1} H^{1 / 2}=\left(\begin{array}{cc}
H_{1}^{1 / 2} P_{1}^{-1} H_{1}^{1 / 2} & 0 \\
-H_{2}^{1 / 2} P_{2}^{-1} B P_{1}^{-1} H_{1}^{1 / 2} & H_{2}^{1 / 2} P_{2}^{-1} H_{2}^{1 / 2}
\end{array}\right) \\
&\left\|H^{1 / 2} P_{L}^{-1} H^{1 / 2}\right\|_{l_{2}} \leq\left\|H_{1}^{1 / 2} P_{1}^{-1} H_{1}^{1 / 2}\right\|_{l_{2}}+\left\|H_{2}^{1 / 2} P_{2}^{-1} B P_{1}^{-1} H_{1}^{1 / 2}\right\|_{l_{2}}+\left\|H_{2}^{1 / 2} P_{2}^{-1} H_{2}^{1 / 2}\right\|_{l_{2}} \\
&=\left\|P_{1}^{-1} H_{1}\right\|_{H_{1}}+\left\|P_{2}^{-1} B P_{1}^{-1}\right\|_{H_{1}^{-1}, H_{2}}+\left\|P_{2}^{-1} H_{2}\right\|_{H_{2}} \\
& \leq \gamma_{1}+\gamma_{2}+\left\|P_{1}^{-1}\right\|_{H_{1}^{-1}, H_{1}}\|B\|_{H_{1}, H_{2}^{-1}}\left\|P_{2}^{-1}\right\|_{H_{2}^{-1}, H_{2}} \\
& \leq \gamma_{1}+\gamma_{2}+\gamma_{1} \gamma_{2} C_{1} .
\end{aligned}
$$

The proof for $P_{R}$ follows in a similar fashion.

\subsection{FOV-equivalent preconditioners}

In this section we consider only two block preconditioners of the form

$$
P_{L}=\left(\begin{array}{cc}
P_{1} & 0 \\
B & -\rho^{-1} P_{2}
\end{array}\right), \quad P_{R}=\left(\begin{array}{cc}
P_{1} & B^{t} \\
0 & -\rho^{-1} P_{2}
\end{array}\right)
$$

As before, if we wish to establish $F O V$-equivalence of our preconditioners to the system matrix we require a slightly stronger version of the coercivity requirements. In particular, 
we still assume that (1.2) hold but also require that the coercivity condition (3.2) hold for F

As before, let $S=B F^{-1} B^{t}$ denote the Schur complement of $F$ in $K$. Assuming we can invert $F$ exactly we obtain the following characterization of $F O V$-equivalence for the above preconditioners.

Theorem 11 Let (1.2), (3.2) hold. Let $P_{L}$ be defined as in (3.4) with $P_{1}=F$. If

$$
P_{2}^{-1} \approx_{H_{2}} S^{-1}
$$

then there exists $\rho_{0}>0$ such that

$$
P_{L}^{-1} \approx_{H} K^{-1}
$$

for all $\rho \geq \rho_{0}$.

Proof Since $P_{2}^{-1} \approx_{H_{2}} S^{-1}$ there exist constants $\beta_{1}, \beta_{2}$ such that

$$
\beta_{1} \leq \frac{\left\langle\mathbf{x}, P_{2}^{-1} S\right\rangle_{H_{2}}}{\langle\mathbf{x}, \mathbf{x}\rangle_{H_{2}}} \leq \beta_{2} .
$$

Since

$$
P_{L}^{-1} K=\left(\begin{array}{ll}
I & F^{-1} B^{t} \\
0 & \rho P_{2}^{-1} S
\end{array}\right)
$$

we need to show that

$\gamma\left(\begin{array}{l}\mathbf{x} \\ \mathbf{y}\end{array}\right)^{t}\left(\begin{array}{cc}H_{1} & 0 \\ 0 & H_{2}\end{array}\right)\left(\begin{array}{l}\mathbf{x} \\ \mathbf{y}\end{array}\right) \leq\left(\begin{array}{l}\mathbf{x} \\ \mathbf{y}\end{array}\right)^{t}\left(\begin{array}{cc}H_{1} & H_{1} F^{-1} B^{t} \\ 0 & \rho H_{2} P_{2}^{-1} S\end{array}\right)\left(\begin{array}{l}\mathbf{x} \\ \mathbf{y}\end{array}\right) \leq \Gamma\left(\begin{array}{l}\mathbf{x} \\ \mathbf{y}\end{array}\right)^{t}\left(\begin{array}{cc}H_{1} & 0 \\ 0 & H_{2}\end{array}\right)\left(\begin{array}{l}\mathbf{x} \\ \mathbf{y}\end{array}\right)$.

Now, the results of Lemmas 7, 8 give

$$
\begin{aligned}
\left\|H_{1} F^{-1} B^{t}\right\|_{H_{2}, H_{1}^{-1}} & \leq\left\|B^{t}\right\|_{H_{2}, H_{1}^{-1}}\left\|H_{1} F^{-1}\right\|_{H_{1}^{-1}, H_{1}^{-1}} \\
& \leq C_{1} C_{3}^{-1} .
\end{aligned}
$$

Hence, using (2.5) we get for all $\mathbf{x} \in \mathbb{R}^{n_{1} \times n_{1}}, \mathbf{y} \in \mathbb{R}^{n_{2} \times n_{2}}$

$$
\left|\mathbf{x}^{t} H_{1} F^{-1} B^{t} \mathbf{y}\right| \leq C_{1} C_{3}^{-1}\|\mathbf{x}\|_{H_{1}}\|\mathbf{y}\|_{H_{2}} .
$$

Thus, using (3.5), (3.6) we obtain

$$
\mathbf{x}^{t} H_{1} \mathbf{x}+\mathbf{x}^{t} H_{1} F^{-1} B^{t} \mathbf{y}+\rho \mathbf{y}^{t} H_{2} P_{2}^{-1} S \mathbf{y} \leq \mathbf{x}^{t} H_{1} \mathbf{x}+C_{1} C_{3}^{-1}\|\mathbf{x}\|_{H_{1}}\|\mathbf{y}\|_{H_{2}}+\rho \beta_{2}\|\mathbf{y}\|_{H_{2}}^{2}
$$

Thus, the upper bound follows immediately with $\Gamma^{2}=\max \left\{1, \rho \beta_{2}\right\}+\frac{1}{2} C_{1} C_{3}^{-1}$.

To obtain the lower bound we set $\mathbf{z}^{t}=\left(\begin{array}{ll}\mathbf{x}^{t} & \mathbf{y}^{t}\end{array}\right)$ and note that (using (3.5), (3.6), $)$

$$
\begin{aligned}
\mathbf{z}^{t} H P_{L}^{-1} K \mathbf{z} & \geq\|\mathbf{x}\|_{H_{1}}^{2}+\rho \beta_{1}\|\mathbf{y}\|_{H_{2}}^{2}-\left|\mathbf{x}^{t} H_{1} F^{-1} B^{t} \mathbf{y}\right| \\
& \geq\|\mathbf{x}\|_{H_{1}}^{2}+\rho \beta_{1}\|\mathbf{y}\|_{H_{2}}^{2}-C_{1} C_{3}^{-1}\|\mathbf{x}\|_{H_{1}}\|\mathbf{y}\|_{H_{2}} \\
& \geq \frac{1}{2}\left(\|\mathbf{x}\|_{H_{1}}^{2}+\|\mathbf{y}\|_{H_{2}}^{2}\right)
\end{aligned}
$$

provided $\rho \geq \rho_{0}:=\frac{1+\left(C_{1} C_{3}^{-1}\right)^{2}}{2 \beta_{1}}$. 
A similar result holds for $P_{R}$.

Theorem 12 Let (1.2), (3.2) hold. Let $P_{R}$ be defined as in (3.4) with $P_{1}=F$. If

$$
S \approx_{H_{2}^{-1}} P_{2}
$$

then there exists $\rho_{0}>0$ such that

$$
K \approx_{H^{-1}} P_{R}
$$

for all $\rho \geq \rho_{0}$.

The previous two theorems indicate that if the inverse of $F$ is available all that is necessary is to approximate the Schur complement in the sense of $F O V$-equivalence. However, the inverse of $F$ may not be readily available in practice and its action may need to be approximated. The subsequent discussion considers this possibility.

For the general choices of preconditioners $P_{L}, P_{R}$ defined in (3.4) the preconditioned system matrix becomes

$$
P_{L}^{-1} K=\left(\begin{array}{cc}
P_{1}^{-1} F & P_{1}^{-1} B^{t} \\
\rho P_{2}^{-1} B\left(P_{1}^{-1} F-I\right) & \rho P_{2}^{-1} \widehat{S}
\end{array}\right), \quad K P_{R}^{-1}=\left(\begin{array}{cc}
F P_{1}^{-1} & \rho\left(F P_{1}^{-1}-I\right) B^{t} P_{2}^{-1} \\
B P_{1}^{-1} & \rho \widehat{S} P_{2}^{-1}
\end{array}\right)
$$

where $\widehat{S}=B P_{1}^{-1} B^{t}$.

Theorem 13 Let (1.2), (3.2) hold. Let $P_{L}$ be defined as in (3.4) and let

$$
P_{1}^{-1} \approx_{H_{1}} F^{-1}, \quad P_{2}^{-1} \approx_{H_{2}} \widehat{S}^{-1} .
$$

Then there exists $\rho_{0}>0$ such that

$$
P_{L}^{-1} \approx_{H} K^{-1}
$$

for all $\rho \geq \rho_{0}$ provided

$$
\left\|P^{-1} F-I\right\|_{H_{1}} \leq 1 / \rho_{0} .
$$

Proof Since $P_{1}^{-1} \approx_{H_{1}} F^{-1}, P_{2}^{-1} \approx_{H_{2}} \widehat{S}^{-1}$, there exist constants $\alpha_{1}, \alpha_{2}, \beta_{1}, \beta_{2}$ such that

$$
\alpha_{1} \leq \frac{\left\langle\mathbf{x}, P_{1}^{-1} F \mathbf{x}\right\rangle_{H_{1}}}{\langle\mathbf{x}, \mathbf{x}\rangle_{H_{1}}}, \quad\left\|P_{1}^{-1} F\right\|_{H_{1}} \leq \alpha_{2}, \quad \beta_{1} \leq \frac{\left\langle\mathbf{x}, P_{2}^{-1} \widehat{S} \mathbf{x}\right\rangle_{H_{2}}}{\langle\mathbf{x}, \mathbf{x}\rangle_{H_{2}}},\left\|P_{2}^{-1} \widehat{S}\right\|_{H_{2}} \leq \beta_{2} .
$$

On the other hand, the equivalence $P_{1}^{-1} \approx_{H_{1}} F^{-1}$ implies that $\widehat{S} \approx_{H_{2}^{-1}} H_{2}$ (Lemma 9) and hence $P_{2} \sim_{H_{2}^{-1}} H_{2}$ by transitivity; in particular,

$$
\left\|P_{2}^{-1}\right\|_{H_{2}^{-1}, H_{2}} \leq \beta_{2} C_{1}^{2} C_{3}^{-1} \quad \text { (cf. Lemma 8). }
$$


We prove the result for the preconditioned system matrix given in (3.7) with $\rho=\rho_{0}$, with $\rho_{0}$ to be specified later. The result will follow for all $\rho \geq \rho_{0}$ in an obvious way. We need to establish the following bounds

$$
\gamma \leq \frac{\left(\begin{array}{l}
\mathbf{x} \\
\mathbf{y}
\end{array}\right)^{t}\left(\begin{array}{cc}
H_{1} P_{1}^{-1} F & H_{1} P_{1}^{-1} B^{t} \\
\rho_{0} H_{2} P_{2}^{-1} B\left(P_{1}^{-1} F-I\right) & \rho_{0} H_{2} P_{2}^{-1} \widehat{S}
\end{array}\right)\left(\begin{array}{l}
\mathbf{x} \\
\mathbf{y}
\end{array}\right)}{\left(\begin{array}{l}
\mathbf{x} \\
\mathbf{y}
\end{array}\right)^{t}\left(\begin{array}{cc}
H_{1} & 0 \\
0 & H_{2}
\end{array}\right)\left(\begin{array}{l}
\mathbf{x} \\
\mathbf{y}
\end{array}\right)} \leq \Gamma
$$

We have

$$
\begin{aligned}
\rho_{0}\left\|H_{2} P_{2}^{-1} B\left(P_{1}^{-1} F-I\right)\right\|_{H_{1}, H_{2}^{-1}} & \leq \rho_{0}\left\|\left(P_{1}^{-1} F-I\right)\right\|_{H_{1}}\|B\|_{H_{1}, H_{2}^{-1}}\left\|H_{2} P_{2}^{-1}\right\|_{H_{2}^{-1}} \\
& \leq C_{1}\left\|P_{2}^{-1}\right\|_{H_{2}^{-1}, H_{2}} \\
& \leq \beta_{2} C_{1}^{3} C_{3}^{-1},
\end{aligned}
$$

where we used $\left\|\left(P_{1}^{-1} F-I\right)\right\|_{H_{1}} \leq 1 / \rho_{0}$. Thus,

$$
\rho_{0}\left|\mathbf{y}^{t} H_{2} P_{2}^{-1} B\left(P_{1}^{-1} F-I\right) \mathbf{x}\right| \leq \beta_{2} C_{1}^{3} C_{3}^{-1}\|\mathbf{x}\|_{H_{1}}\|\mathbf{y}\|_{H_{2}}
$$

Similarly we find

$$
\begin{aligned}
\left\|H_{1} P_{1}^{-1} B^{t}\right\|_{H_{2}, H_{1}^{-1}} & \leq\left\|B^{t}\right\|_{H_{2}, H_{1}^{-1}}\left\|F^{-1}\right\|_{H_{1}^{-1}, H_{1}}\left\|P_{1}^{-1} F\right\|_{H_{1}, H_{1}}\left\|H_{1}\right\|_{H_{1}, H_{1}^{-1}} \\
& \leq C_{1} C_{3}^{-1} \alpha_{2}
\end{aligned}
$$

so that

$$
\left|\mathbf{x}^{t} H_{1} P_{1}^{-1} B^{t} \mathbf{y}\right| \leq C_{1} C_{3}^{-1} \alpha_{2}\|\mathbf{x}\|_{H_{1}}\|\mathbf{y}\|_{H_{2}}
$$

and since $\left\|P_{2}^{-1} \widehat{S}\right\|_{H_{2}} \leq \beta_{2}$,

$$
\left|\mathbf{y}^{t} H_{2} P_{2}^{-1} \widehat{S} \mathbf{y}\right| \leq \beta_{2}\|\mathbf{y}\|_{H_{2}}^{2} .
$$

Setting $\mathbf{z}^{t}=\left(\mathbf{x}^{t}, \mathbf{y}^{t}\right)$ we have

$\mathbf{z}^{t} H P_{L}^{-1} K \mathbf{z}=\mathbf{x}^{t} H_{1} P_{1}^{-1} F \mathbf{x}+\mathbf{x}^{t} H_{1} P_{1}^{-1} B^{t} \mathbf{y}+\rho_{0} \mathbf{y}^{t} H_{2} P_{2}^{-1} B\left(P_{1}^{-1} F-I\right) \mathbf{x}+\rho_{0} \mathbf{y}^{t} H_{2} P_{2}^{-1} \widehat{S} \mathbf{y}$

and therefore

$$
\mathbf{z}^{t} H P_{L}^{-1} K \mathbf{z} \leq \alpha_{2}\|\mathbf{x}\|_{H_{1}}^{2}+\left(C_{1} C_{3}^{-1} \alpha_{2}+\beta_{2} C_{1}^{3} C_{3}^{-1}\right)\|\mathbf{x}\|_{H_{1}}\|\mathbf{y}\|_{H_{2}}+\rho_{0} \beta_{2}\|\mathbf{y}\|_{H_{2}}^{2}
$$

and

$$
\mathbf{z}^{t} H P_{L}^{-1} K \mathbf{z} \geq \alpha_{1}\|\mathbf{x}\|_{H_{1}}^{2}-\left(C_{1} C_{3}^{-1} \alpha_{2}+\beta_{2} C_{1}^{3} C_{3}^{-1}\right)\|\mathbf{x}\|_{H_{1}}\|\mathbf{y}\|_{H_{2}}+\rho_{0} \beta_{1}\|\mathbf{y}\|_{H_{2}}^{2}
$$

Hence, the lower bound follows with $\gamma=\frac{1}{2} \alpha_{1}$, provided we take $\rho_{0}=\frac{\left(C_{1} C_{3}^{-1} \alpha_{2}+\beta_{2} C_{1}^{3} C_{3}^{-1}\right)^{2}+\alpha_{1}^{2}}{2 \alpha_{1} \beta_{1}}$ and the upper bound follows with $\Gamma^{2}=\max \left\{\alpha_{2}, \rho_{0} \beta_{2}\right\}+\frac{1}{2}\left(C_{1} C_{3}^{-1} \alpha_{2}+\beta_{2} C_{1}^{3} C_{3}^{-1}\right)$.

Finally, we have a similar result for the right preconditioner $P_{R}$. 
Theorem 14 Let (1.2), (3.2) hold. Let $P_{R}$ be defined as in (3.4) and let

$$
F \approx_{H_{1}^{-1}} P_{1}, \quad \widehat{S} \approx_{H_{2}^{-1}} P_{2} .
$$

Then there exists $\rho_{0}>0$ such that

$$
K \approx_{H^{-1}} P_{R}
$$

for all $\rho \geq \rho_{0}$ provided

$$
\left\|F P_{1}^{-1}-I\right\|_{H_{1}^{-1}} \leq 1 / \rho_{0}
$$

\section{Applications}

The analysis of section 3 was motivated by the need to describe convergence for nonsymmetric preconditioned techniques for saddle-point problems; let us start, though, with some remarks concerning the symmetric case.

When $K$ is symmetric, an optimal preconditioner is the matrix norm (or some approximation of it) as described by the previous theory. This is the preconditioner considered in all applications we know of. Moreover, since MINRES is the solver of choice for symmetric indefinite problems, the analyses presented in the literature address the distribution of eigenvalues of the symmetrically preconditioned system $H^{-1 / 2} K H^{-1 / 2}$. This is contained in our analysis in the form of Lemma 4 which showed that

$$
\begin{aligned}
\left\|H^{-1 / 2} K H^{-1 / 2}\right\|_{l_{2}}=\left\|H^{-1} K\right\|_{H} & \leq C_{1} . \\
\left\|H^{1 / 2} K^{-1} H^{1 / 2}\right\|_{l_{2}}=\left\|H K^{-1}\right\|_{H^{-1}} & \leq C_{2}^{-1} .
\end{aligned}
$$

By Remark 2, the moduli of the eigenvalues are bounded from below and above by $C_{2}$ and $C_{1}$, respectively. Thus, a general convergence result takes the form (cf. (2.8))

$$
\frac{\left\|\widehat{\mathbf{r}}^{k}\right\|_{l_{2}}}{\left\|\widehat{\mathbf{r}}^{0}\right\|_{l_{2}}}=\frac{\left\|\mathbf{r}^{k}\right\|_{H^{-1}}}{\left\|\mathbf{r}^{0}\right\|_{H^{-1}}} \leq 2\left(\frac{C_{1}-C_{2}}{C_{1}+C_{2}}\right)^{k / 2}
$$

where $\widehat{\mathbf{r}}^{k}=H^{-1 / 2} \mathbf{r}^{k}$. Thus, convergence is independent of $n$ and is measured in the natural norm, $\|\cdot\|_{H^{-1}}$. This, or similar results appear in almost all analyses of preconditioners for symmetric and indefinite saddle-point problems [7], [42], [5], [43], [41], [8], [4] etc. We refer the reader to these references for numerical results.

\subsection{Example: Navier-Stokes flow}

The application we investigate in this section comes from the Picard linearization of the Navier-Stokes equations. The formulation of the problems is as follows.

Let $\Omega \subset \mathbb{R}^{2}$ be a bounded domain with boundary $\Gamma$. The Navier-Stokes equations with Dirichlet boundary conditions read

$$
\begin{aligned}
-\nu \Delta \mathbf{u}+(\mathbf{u} \cdot \nabla) \mathbf{u}+\nabla p & =\mathbf{f} & & \text { in } \Omega \\
\div \mathbf{u} & =0 & & \text { in } \Omega, \\
\mathbf{u} & =\mathbf{u}^{*} & & \text { on } \Gamma,
\end{aligned}
$$


where $\nu$ is the viscosity parameter, $\mathbf{u}$ is the velocity and $p$ the pressure.

A simple solution method involves linearizing (4.1) using a Picard, or fixed point, iteration. This leads to a sequence of linear problems; thus, at each Picard step we need to solve an Oseen problem of the form

$$
\begin{aligned}
-\nu \Delta \mathbf{u}+(\mathbf{b} \cdot \nabla) \mathbf{u}+\nabla p & =\mathbf{f} & & \text { in } \Omega, \\
\div \mathbf{u} & =0 & & \text { in } \Omega, \\
\mathbf{u} & =\mathbf{u}^{*} & & \text { on } \Gamma,
\end{aligned}
$$

where $\mathbf{b}$ is the approximation to $\mathbf{u}$ obtained in the previous Picard step.

A mixed formulation of problem (4.2) involves choosing appropriate spaces

$$
\mathbf{V} \subset\left[H_{E}^{1}(\Omega)\right]^{2}=\left\{\boldsymbol{\phi} \in\left[H^{1}(\Omega)\right]^{2}:\left.\boldsymbol{\phi}\right|_{\Gamma}=\mathbf{0}\right\}, P \subset L_{0}^{2}(\Omega)=\left\{p \in L^{2}(\Omega):\langle p, 1\rangle=0\right\}
$$

for the velocity and pressure respectively and results in the following weak formulation

$$
\text { Given } \mathbf{f} \in\left[L^{2}(\Omega)\right]^{2} \text {, find }(\mathbf{u}, p) \in \mathcal{H}=\mathbf{V} \times P \text { such that }
$$

$$
\mathcal{K}(\mathbf{u}, p ; \mathbf{v}, q)=\mathcal{F}(\mathbf{v}, q) \quad \forall(\mathbf{v}, q) \in \mathcal{H}
$$

where

$$
\mathcal{K}(\mathbf{w}, r ; \mathbf{v}, q)=\nu\langle\nabla \mathbf{w}, \nabla \mathbf{v}\rangle+\langle\mathbf{b} \cdot \nabla \mathbf{w}, \mathbf{v}\rangle-\langle r, \div \mathbf{v}\rangle-\langle q, \div \mathbf{w}\rangle
$$

and $\mathcal{F}$ is a linear functional on $\mathcal{H}$ given by

$$
F(\mathbf{v}, q)=\langle\mathbf{f}, \mathbf{v}\rangle .
$$

Existence and uniqueness follow from the continuity and coercivity conditions of Babuška with respect to a suitable norm $\|\cdot\|_{\mathcal{H}}$

$$
\begin{gathered}
|\mathcal{K}(\mathbf{w}, r ; \mathbf{v}, q)| \leq C_{1}\|\mathbf{w}, r\|_{\mathcal{H}}\|\mathbf{v}, q\|_{\mathcal{H}} \\
\sup _{(\mathbf{w}, r) \in \mathcal{H} \backslash\{0\}} \frac{\mathcal{K}(\mathbf{w}, r ; \mathbf{v}, q)}{\|\mathbf{w}, r\|_{\mathcal{H}}} \geq C_{2}\|\mathbf{v}, q\|_{\mathcal{H}} \\
\sup _{(\mathbf{v}, q) \in \mathcal{H} \backslash\{0\}} \frac{\mathcal{K}(\mathbf{w}, r ; \mathbf{v}, q)}{\|\mathbf{v}, q\|_{\mathcal{H}}} \geq C_{2}\|\mathbf{w}, r\|_{\mathcal{H}}
\end{gathered}
$$

which lead to conditions (1.2). In particular, it can be shown that conditions (1.2) hold with respect to the discrete norm

$$
\left\|\mathbf{v}^{h}, p^{h}\right\|_{H}^{2}=\nu\left\|\nabla \mathbf{v}^{h}\right\|_{L^{2}(\Omega)}^{2}+\left\|p^{h}\right\|_{L^{2}(\Omega)}^{2} \quad\left(\mathbf{v}^{h}, p^{h}\right) \in \mathcal{H}_{h} \subset \mathcal{H}
$$

provided $\mathcal{H}_{h}=\mathbf{V}^{h} \times P^{h}$ is a suitable pair of finite element spaces. This choice of spaces leads to a linear system of equations with coefficient matrix of type (1.3); the corresponding discrete matrix-norms are a scaled discrete Laplacian $\left(H_{1}\right)$ and a mass matrix $\left(H_{2}\right)$. 
Block-preconditioning techniques for this problem have already been considered in the literature [17], [31], [16], [18], [29], [33]. We discuss below some of the existing preconditioners and the corresponding analyses.

Elman and Silvester were the first to suggest in [17] block-preconditioners of type (3.4) where $P_{1}=F$ and $P_{2}=H_{2} / \nu$. Their analysis shows that the eigenvalues are in the left-half plane and that they are independent of the size of the problem $n$. The same preconditioner was considered by Klawonn and Starke in [31]. Their analysis improved that of [17]; in particular, they showed that the $H$-field-of-values lies in the right-half plane and is independent of $n$. All these results are confirmed by our analysis. In particular, Thms 11, 12 show that both the $H$ - and the $H^{-1}$-fields of values are independent of $n$ and lie in the right-half plane so that GMRES will converge with respect to the $H$ - and respectively $H^{-1}$-norms with a convergence bound given by (2.9).

The above preconditioner, although mesh-independent in performance, has a dependence of order $O\left(\nu^{-1}\right)$ on the number of iterations. Moreover, the mesh-independence 'sets in' at a relatively high number of iterations. This is explained by the fact that the preconditioning technique essentially tries to approximate a non-symmetric Schur complement with a symmetric preconditioner $\left(H_{2} / \nu\right)$. As $\nu$ reduces, the problem becomes more nonsymmetric and the bound (2.9) deteriorates. An improved preconditioner of type (3.4) was introduced in [29]. As before, $P_{1}=F$, but $P_{2}$ is assembled on the pressure finite element space $P^{h}$ and is defined via

$$
P_{2}^{-1}=H_{2}^{-1} F_{p} A_{p}^{-1}
$$

where $F_{p}, A_{p}$ are the projections of the advection-diffusion and diffusion operators respectively onto the finite-element space. This preconditioner was analyzed in [33] where it is shown that the Schur complement is $l_{2}$-norm equivalent to $P_{2}$. Since in this case the $l_{2}$ and $\mathrm{H}_{2}$-norms are equivalent, there holds

$$
S \sim_{H_{2}^{-1}} P_{2}, \quad P_{2}^{-1} \sim_{H_{2}} S^{-1}
$$

so that we can apply Thm 10 to find that

$$
K \sim_{H^{-1}} P_{R}, \quad P_{L}^{-1} \sim_{H} K^{-1}
$$

This result implies that the eigenvalues are bounded independently of $n$. Numerical results in the next section indicate that convergence also is bounded independently of $n$; moreover, the dependence on the viscosity parameter is improved to $O\left(\nu^{-1 / 2}\right)$. However, more descriptive GMRES bounds than (2.9) are necessary to account for improvements in behaviour with respect to parameters other than $n$ (see also [18] for an eigenvalue analysis for this problem).

\subsection{Numerical experiments}

Our analysis indicates that convergence is independent of the size of the problem provided we have equivalent preconditioners and we work in a suitable norm. Preconditioning from 
the left requires the $H$-norm, which is not expensive to implement; however, right preconditioning requires the $H^{-1}$-norm, which has to be approximated somehow. In the numerical experiments below we implement GMRES in both norms as well as in the standard $l_{2}$ norm. It turns out that convergence is similar in all three cases. While it is not obvious why the three versions of GMRES should be equivalent, we can use this empirical result to infer that our analysis leads to $l_{2}$-equivalent preconditioners. This is also consistent with the analytic results in [34], [23] and the experiments in [2], where the same equivalence is shown to hold for the case of scalar elliptic problems.

The test problem we chose is the regularized driven-cavity problem. The domain is the unit square with zero boundary conditions except for $\mathbf{u}^{*}(x, y=1)=16 x^{2}(1-x)^{2}$ (cf. $(4.2 \mathrm{c}))$. The problem was solved for a range of viscosity parameters $\nu$ and mesh parameters $h$. The preconditioners we used were of type (3.4). The stopping criterion at each Picard step was of the form

$$
\frac{\left\|\mathbf{r}^{k}\right\|_{*}}{\left\|\mathbf{r}^{0}\right\|_{*}} \leq \tau,
$$

where $\|\cdot\|_{*}$ was the norm induced by the inner-product in which we implemented GMRES; $\tau=10^{-3}$ was sufficient to advance the Picard iteration.

We first implemented a norm-equivalent preconditioner given by the choice $P_{1}=F$ and $P_{2}$ as defined above in (4.6). Results for GMRES with respect to the inner-products $\langle\cdot, \cdot\rangle_{l_{2}},\langle\cdot, \cdot\rangle_{H}$ with left preconditioning are shown in Tables 1,2 , respectively. As expected, the performance is indeed independent of the size of the problem. Furthermore, as mentioned above, the results are strikingly similar. The use of a different inner-product does not seem to affect the convergence of GMRES.

\begin{tabular}{r|cccccc}
$\nu=$ & $1 / 10$ & $1 / 20$ & $1 / 40$ & $1 / 80$ & $1 / 160$ & $1 / 320$ \\
\hline$n=2,467$ & 13.2 & 11.0 & 13.7 & 13.0 & 16.6 & 19.6 \\
9,539 & 14.0 & 11.8 & 12.2 & 13.3 & 16.1 & 19.2 \\
37,507 & 11.8 & 8.8 & 10.0 & 11.3 & 13.4 & 17.0
\end{tabular}

Table 1: GMRES in the $l_{2}$-inner product with left preconditioning: average iteration count over the Picard steps.

\begin{tabular}{r|cccccc}
$\nu=$ & $1 / 10$ & $1 / 20$ & $1 / 40$ & $1 / 80$ & $1 / 160$ & $1 / 320$ \\
\hline$n=2,467$ & 11.8 & 11.4 & 13.8 & 15.8 & 17.0 & 22.1 \\
9,539 & 15.0 & 12.5 & 12.7 & 13.4 & 16.5 & 19.5 \\
37,507 & 13.0 & 9.3 & 10.2 & 11.3 & 13.7 & 17.1
\end{tabular}

Table 2: GMRES in the $H$-inner product with left preconditioning: average iteration count over the Picard steps.

Similar results were obtained for GMRES with respect to the inner-products $\langle\cdot, \cdot\rangle_{l_{2}},\langle\cdot, \cdot\rangle_{H}$ and right preconditioning as shown in Tables 3,4 . 


\begin{tabular}{r|cccccc}
$\nu=$ & $1 / 10$ & $1 / 20$ & $1 / 40$ & $1 / 80$ & $1 / 160$ & $1 / 320$ \\
\hline$n=2,467$ & 16.0 & 12.4 & 17.6 & 19.9 & 29.0 & 37.4 \\
9,539 & 12.0 & 15.4 & 17.8 & 22.7 & 24.5 & 38.2 \\
37,507 & 12.5 & 14.25 & 19.0 & 23.16 & 28.7 & 34.1
\end{tabular}

Table 3: GMRES in the $l_{2}$-inner product with right preconditioning: average iteration count over the Picard steps.

\begin{tabular}{r|cccccc}
$\nu=$ & $1 / 10$ & $1 / 20$ & $1 / 40$ & $1 / 80$ & $1 / 160$ & $1 / 320$ \\
\hline$n=2,467$ & 14.8 & 18.8 & 20.2 & 21.9 & 27.3 & 36.9 \\
9,539 & 15.5 & 18.2 & 18.2 & 21.4 & 25.4 & 31.1 \\
37,507 & 16.25 & 15 & 16 & 23.3 & 24.0 & 33.5
\end{tabular}

Table 4: GMRES in the $H^{-1}$-inner product with right preconditioning: average iteration count over the Picard steps.

\section{Conclusions}

We presented a general analysis of block-preconditioning techniques for mixed finite element formulations of saddle-point problems. The analysis requires as hypothesis only the stability conditions of the mixed finite element formulation. The theoretical results confirm previous work in the literature and provide a tool for the design and analysis of preconditioners for other problems which afford a similar formulation. Moreover, our numerical experiments highlight the fact that, while the analysis is performed with respect to the natural norm of the problem, standard $\left(l_{2}\right)$ implementations of GMRES appear to be equivalent. While it is not clear why such a remarkable equivalence should hold, we can nonetheless use it to our advantage to deduce that optimality with respect to the natural norm holds also with respect to the standard $l_{2}$-norm. We hope to address this issue in future research.

\section{References}

[1] M. Arioli. A stopping criterion for the conjugate gradient algorithm in a finite element method framework. Technical Report IAN-1179, Istituto di Analisi Numerica, Pavia, 2000 .

[2] M. Arioli, D. Loghin, and A. J. Wathen. A note on stopping criteria for finite element methods. In preparation, 2002.

[3] M. Arioli, E. Noulard, and A. Russo. Stopping criteria for iterative methods: applications to PDEs. Calcolo, 38:97-112, 2001.

[4] D. N. Arnold, R. S. Falk, and R. Winther. Preconditioning discrete approximations of the Reissner-Mindlin plate model. RAIRO Model. Math. Anal. Numer., 31(4):517-557, 1997. 
[5] D. N. Arnold, R. S. Falk, and R. Winther. Preconditioning in $H(d i v)$ and applications. Math. Comp., 66(219):957-984, 1997.

[6] I. Babuška. Error bounds for finite element methods. Numer. Math., 16:322-333, 1971.

[7] J. H. Bramble and J. E. Pasciak. A preconditioning technique for indefinite systems resulting from mixed approximations of elliptic problems. Math. Comp., 50:1-17, 1988.

[8] J. H. Bramble and J. E. Pasciak. Iterative techniques for time dependent Stokes problems. Computers Math. Applic., 33(1-2):13-30, 1997.

[9] F. Brezzi. On the existence, uniqueness and approximation of saddle-point problems arising from Lagrangian multipliers. RAIRO Anal. Numer., 8:129-151, 1974.

[10] F. Brezzi and K. J. Bathe. A discourse on the stability conditions for mixed finite element formulations. Comp. Meth. Appl. Mech. Engrg., 82:27-57, 1990.

[11] B. M. Brown, P. K. Jimack, and M. D. Mihajlovic. An efficient direct solver for a class of mixed finite element problems. Applied Numerical Mathematics, 38:1-20, 2000.

[12] J. Cahouet and J. P. Chabard. Some fast 3D finite element solvers for the generalized Stokes problem. Internat. J. Numer. Methods Fluids, 8(8):869-895, 1988.

[13] H. H. Chen and J. C. Strikwerda. Preconditioning for regular elliptic systems. SIAM J. Num. Anal., 37(1):131-151, 1999.

[14] Z. Chen, R. E. Ewing, and R. Lazarov. Domain decomposition algorithms for mixed methods for second order elliptic problems. Math. Comp., 65:467-490, 1996.

[15] H. C. Elman. Iterative methods for large sparse non-symmetric systems of linear equations. PhD thesis, Yale University, New Haven, 1982.

[16] H. C. Elman. Preconditioning for the steady-state Navier-Stokes equations with low viscosity. SIAM J. Sci. Comp., 20(4):1299-1316, 1999.

[17] H. C. Elman and D. J. Silvester. Fast nonsymmetric iterations and preconditioning for Navier-Stokes equations. SIAM J. Sci. Comp., 17:33-46, 1996.

[18] H. C. Elman, D. J. Silvester, and A. J. Wathen. Performance and analysis of saddle point preconditioners for the discrete steady-state Navier-Stokes equations. Numer. Math., 90(4):665-688, 2001.

[19] V. Faber, T. A. Manteuffel, and S. V. Parter. On the theory of equivalent operators and application to the numerical solution of uniformly elliptic partial differential equations. Adv. Appl. Math., 11:109-163, 1990.

[20] B. Fischer, A. J. Wathen, and D. J. Silvester. The convergence of iterative solution methods for symmetric and indefinite linear systems. In Numerical analysis 1997 (Dundee), pages 230-243. Longman, Harlow, 1998. 
[21] R. Glowinski and O. Pironneau. Numerical methods for the first biharmonic equation and for the two-dimensional Stokes problem. SIAM Rev., 21(2):167-212, 1979.

[22] R. Glowinski and M. Wheeler. Domain decomposition and mixed finite element methods for elliptic problems. In R. Glowinski et. al., editor, First International Symposium on Domain Decomposition Methods for Partial Differential Equations, pages 144-172. SIAM, Philadelphia, 1988.

[23] C. I. Goldstein, T. A. Manteuffel, and S. V. Parter. Preconditioning and boundary conditions without $H_{2}$ estimates: $L_{2}$ condition numbers and the distribution of the singular values. SIAM J. Num. Anal., 30(2):343-376, 1993.

[24] G. H. Golub and G. Meurant. Matrices, moments and quadrature II; how to compute the norm of the error in iterative methods. BIT, 37(3):687-705, 1997.

[25] G. H. Golub and Z. Strakoš. Estimates in quadratic formulas. Numer. Algorithms, 8:2-4, 1994.

[26] A. Greenbaum. Iterative methods for solving linear systems. SIAM, 1997.

[27] R. A. Horn and C. R. Johnson. Matrix Analysis. Cambridge University Press, 1985.

[28] D. Kay and D. Loghin. A Green's function preconditioner for the steady-state NavierStokes equations. Technical Report 99/06, Oxford University Computing Laboratory, 1999.

[29] D. Kay, D. Loghin, and A. J. Wathen. A preconditioner for the Steady-State NavierStokes equations. SIAM J. Sci. Comput., accepted for publication 2002.

[30] A. Klawonn. Block-triangular preconditioners for saddle-point problems with a penalty term. SIAM J. Sci. Comput., 19(1):172-184, 1998.

[31] A. Klawonn and G. Starke. Block triangular preconditioners for non-symmetric saddlepoint problems: field-of-values analysis. Numer. Math., 81, 1999.

[32] P. Krzyzanowski. On block preconditioners for nonsymmetric saddle point problems. SIAM J. Sci. Comp., 2001.

[33] D. Loghin. Analysis of preconditioned Picard iterations for the Navier-Stokes equations. Submitted to Numer. Math, 2001.

[34] T. A. Manteuffel and S. V. Parter. Preconditioning and boundary conditions. SIAM J. Num. Anal., 27:656-694, 1989.

[35] G. Meurant. Numerical experiments in computing bounds for the norm of the error in the preconditioned conjugate gradient algorithm. Numer. Algorithms, 22:353-365, 1999. 
[36] M. D. Mihajlovic and D. J. Silvester. Efficient preconditioning of the biharmonic equation. Technical Report 362, UMIST, 2000.

[37] I. Perugia and V. Simoncini. Optimal and quasi-optimal preconditioners for certain mixed finite element approximations. Technical Report 1098, Istituto di Analisi Numerica del C.N.R., Pavia, 1998.

[38] I. Perugia, V. Simoncini, and M. Arioli. Linear algebra methods in a mixed approximation of magnetostatic problems. SIAM J. Sci. Comput., 21(3):1085-1101, 1999.

[39] T. Rusten and R. Winther. Substructure preconditioners for elliptic saddle point problems. Math. Comp., 60(201):23-48, 1993.

[40] Y. Saad. Iterative Methods for Sparse Linear Systems. PWS Publishing Co., Boston, 1996.

[41] D. J. Silvester and A. J. Wathen. Fast iterative solution of stabilised Stokes systems. II. Using general block preconditioners. SIAM J. Num. Anal., 31(5):1352-1367, 1994.

[42] P. S. Vassilevski and J. Wang. Multilevel iterative methods for mixed finite element discretizations of elliptic problems. Numer. Math., 63:503-520, 1992.

[43] A. J. Wathen and D. J. Silvester. Fast iterative solution of stabilised Stokes systems. I. Using simple diagonal preconditioners. SIAM J. Num. Anal., 30(3):630-649, 1993. 